\title{
The optimum decision rules in the same-different paradigm
}

\author{
HUANPING DAI \\ University of Florida, Gainesville, Florida \\ NIEK J. VERSFELD \\ TNO Human Factors Research Institute, Soesterberg, The Netherlands \\ and \\ DAVID M. GREEN \\ University of Florida, Gainesville, Florida
}

\begin{abstract}
In this paper we derive the optimum (likelihood-ratio) decision statistic for a same-different paradigm. The likelihood ratio is dependent on the degree of correlation between the two observations on each trial. For the two extreme cases in which the observations are either independent or highly correlated, the optimum decision rule is identical to each of two previously suggested decision rules. For these two cases, the receiver-operating characteristic (ROC) curves are calculated. Finally, an experimental procedure is suggested for assessing the decision rule actually used by the observer in a samedifferent task.
\end{abstract}

This paper derives the optimum (likelihood-ratio) decision rule for a same-different paradigm. In the samedifferent paradigm there are two types of trials, one having a pair of identical stimuli, and the other having a pair of different stimuli. The subject responds either "same" or "different." Suppose an experimenter applies the samedifferent paradigm to measure a listener's ability to discriminate between two tones having different frequencies of $f_{A}=f-\Delta f$ and $f_{B}=f+\Delta f$. Here $f$ can be regarded as a standard frequency. On each trial, the experimenter may present a pair of tones at frequencies of $\left\langle f_{A}, f_{A}\right\rangle,\left\langle f_{B}, f_{B}\right\rangle$, $\left\langle f_{A}, f_{B}\right\rangle$, or $\left\langle f_{B}, f_{A}\right\rangle$. The correct response is "same" for the first two pairs, and "different" for the last two pairs. In this example, the relevant stimulus cue is the pitch of the tone. Sometimes, however, the relevant stimulus cues are unknown, or the experimenter may have difficulties in attempting to describe the stimulus differences to the subjects (as in wine tasting). In such cases, the same-different task is particularly useful, because the subject can use whatever clues are useful in making the discrimination. Incidentally, another paradigm that is useful for such cases is the oddity paradigm, which is treated in a companion paper by Versfeld, Dai, and Green (1996).

Two decision rules have been suggested previously for the same-different paradigm. Suppose the two observations on a trial are given by the vector $\boldsymbol{\xi}=\left[\xi_{1} \xi_{2}\right]^{T}$, where $T$ denotes transpose - that is, changes a row vector into a column vector, and vice versa - then, according to Sorkin's

The authors are grateful to Todd Maddox, David Noreen, and an anonymous reviewer for their valuable comments, and for their help in making the manuscript easier to follow. Correspondence should be addressed to N. J. Versfeld, TNO Human Factors Research Institute, P. O. Box 23, 3769 ZG Soesterberg, The Netherlands.
(1962) decision rule, the subject should respond "different" only if the absolute difference of the two observations, $\left|\xi_{1}-\xi_{2}\right|$, is greater than some criterion value. The second decision rule is the likelihood-ratio rule derived by Noreen (1981; cf. Macmillan \& Creelman, 1991). According to Noreen's decision rule, the subject should respond "different" only if $\left(\xi_{1}-\mu_{f}\right)\left(\xi_{2}-\mu_{f}\right)<0$ is true. Here $\mu_{f}$ is the (expected value of the) pitch associated with the standard frequency $f$. Macmillan and Creelman (1991) suggested that Noreen's rule is appropriate for conditions using a fixed standard, whereas Sorkin's rule is appropriate for conditions using a variable - or roving — standard. One question addressed by this paper is the relation between these two decision rules.

In this paper, we derive the likelihood-ratio decision statistic assuming an arbitrary correlation between the observations. The concept of correlation can be understood via our example of frequency discrimination. In that experiment, the listener hears two tones having nearly the same frequency. Let us call the first tone $f_{1}$, and the second, $f_{2}$. The observation of those tones can be represented by two random variables, $\xi_{1}$ and $\xi_{2}$. If the standard frequency, $f$, of these two tones is fixed over trials, the covariance of these two random variables is zero by assumption (i.e., the subject's processing can be represented by two random variables that are assumed to be uncorrelated):

$$
\operatorname{COV}\left[\xi_{1}, \xi_{2}\right]=E\left[\left(\xi_{1}-E\left[\xi_{1}\right]\right)\left(\xi_{2}-E\left[\xi_{2}\right]\right)\right]=0
$$

When the standard frequency is randomly changed from trial to trial, both frequencies in the two observation intervals are modified by the same random variable, $r$. Thus we have the observation $\xi_{1}=\zeta_{1}+\omega_{r}$ in the first interval and the observation $\xi_{2}=\zeta_{2}+\omega_{r}$ in the second interval, where $\zeta_{1}$ and $\zeta_{2}$ are independent of each other, and $\omega_{r}$ represents the cor- 
related part. Now the covariance between the two observations is

$$
\begin{aligned}
\operatorname{COV}\left[\xi_{1}, \xi_{2}\right] & =\operatorname{COV}\left[\zeta_{1}+\omega_{r}, \zeta_{2}+\omega_{r}\right] \\
& =E\left[\left(\zeta_{1}+\omega_{r}-E\left[\zeta_{1}+\omega_{r}\right]\right)\left(\zeta_{2}+\omega_{r}-E\left[\zeta_{2}+\omega_{r}\right]\right)\right] \\
& =E\left[\left(\omega_{r}-E\left[\omega_{r}\right]\right)^{2}\right]=\operatorname{VAR}\left[\omega_{r}\right]
\end{aligned}
$$

If the variance of $\omega_{r}$ is large in comparison with the variance of $\zeta_{1}$ or $\zeta_{2}$, the correlation between the two observations in a trial approaches unity. A correlation of near unity is easy to achieve in an experimental situation by making the random change in frequency, $r$, large in comparison with the frequency difference limen.

In this paper, it will be shown that if there is no correlation between the observations (fixed standard), the optimum rule is identical to the rule suggested by Noreen (1981), and if the observations are highly correlated (roving standard), the optimum rule is identical to the rule suggested by Sorkin (1962). Thus, the correlation between observations provides a common thread between the two decision rules. Next, the receiver-operating characteristic (ROC) functions are calculated by assuming each of the decision rules. For a given pair of correct and incorrect "different" response proportions, the $d^{\prime}$ value is different depending on which decision rule is used. Finally, an experimental procedure is suggested for empirically determining which decision rule is used by the observer.

\section{THE LIKELIHOOD-RATIO DECISION STATISTIC}

The two observations on a single trial, $\xi_{1}$ and $\xi_{2}$, can be written as a vector $\boldsymbol{\xi}=\left[\xi_{1} \xi_{2}\right]^{T}$. Assume that $\boldsymbol{\xi}$ has a bivariate Gaussian distribution, with expected values that have an equal probability to be $\boldsymbol{\mu}_{A A}=\left[\mu_{A} \mu_{A}\right]^{T}, \boldsymbol{\mu}_{A B}=\left[\mu_{A} \mu_{B}\right]^{T}$, $\boldsymbol{\mu}_{B A}=\left[\mu_{B} \mu_{A}\right]^{T}$, or $\boldsymbol{\mu}_{B B}=\left[\mu_{B} \mu_{B}\right]^{T}$, where $\mu_{A}$ and $\mu_{B}$ are the expected values of $\xi$, given stimulus presentation $A$ or $B$, respectively. We arbitrarily let $\mu_{B}>\mu_{A}$; their difference, $\mu_{B}-\mu_{A}$, represents the subjective effect of the signal size. The correct response is "same" for the first and fourth expected values, and "different" otherwise. For the purpose of analysis, we introduce $\mu=\left(\mu_{A}+\mu_{B}\right) / 2$. The two observations of each trial have an independent variance of $\sigma^{2}$ and a common variance of $\sigma_{R}^{2}$, so that the total variance of each observation is $\sigma^{2}+\sigma_{R}^{2}$. The correlation coefficient between the two observations is, by definition, $\rho=$ $\sigma_{R}^{2} /\left(\sigma^{2}+\sigma_{R}^{2}\right)$. If we let $d^{\prime}=\left(\mu_{B}-\mu_{A}\right) / \sigma\left(d^{\prime} \geq 0\right)$, we can express the likelihood ratio of the different hypothesis over the same hypothesis (Appendix A) as

$$
\mathcal{L}_{d, s}(\xi)=\exp \left[-\left(\frac{d^{\prime}}{2}\right)^{2}\left(1-\varepsilon^{2}\right)\right] \frac{\cosh \left[\frac{d^{\prime}}{2} \frac{\xi_{2}-\xi_{1}}{\sigma}\right]}{\cosh \left[\frac{d^{\prime}}{2} \varepsilon^{2} \frac{\xi_{1}+\xi_{2}-2 \mu}{\sigma}\right]},
$$

$$
\varepsilon^{2}=\frac{\sigma^{2}}{\sigma^{2}+2 \sigma_{R}^{2}}=\frac{1-\rho}{1+\rho},
$$

or, equivalently,

$$
\rho=\frac{1-\varepsilon^{2}}{1+\varepsilon^{2}} .
$$

Thus if $\sigma_{R}^{2}=0$, then $\rho=0$ and $\varepsilon^{2}=1$, and if $\sigma_{R}^{2} \gg \sigma^{2}$, then $\rho \longrightarrow 1$ and $\varepsilon^{2} \longrightarrow 0$. The optimum decision rule is to respond "different" where $\mathcal{L}_{d, s}(\xi)>C$, and to respond "same" otherwise, where $C$ is the decision criterion. The value of $C$ depends on the a priori probability and the values and costs associated with each stimulus-response alternative. If there is no bias toward either response, we have $C=1$. Clearly, the value of $\mu$ must be known to apply the optimum decision rule.

The expression for the likelihood ratio (Equation 5) may be simplified for two special cases, depending on the value of the correlation between the two observations $\xi_{1}$ and $\xi_{2}$. One case is when the observations are independent - that is, $\sigma_{R}=0(\rho=0)$, or, equivalently, $\varepsilon^{2}=1$-and the other case is when the observations are highly correlated-that is, $\sigma_{R} \gg \sigma\left(\rho \longrightarrow 1\right.$ or $\left.\varepsilon^{2} \longrightarrow 0\right)$.

\section{The Optimum Decision Rule for Independent Observations}

When $\sigma_{R}^{2}=0\left(\varepsilon^{2}=1, \rho=0\right)$, the observations are independent; then Equation 5 reduces to

$$
\mathcal{L}_{d, s}(\xi)=\frac{\cosh \left[\frac{d^{\prime}}{2} \frac{\xi_{2}-\xi_{1}}{\sigma}\right]}{\cosh \left[\frac{d^{\prime}}{2} \frac{\xi_{1}+\xi_{2}-2 \mu}{\sigma}\right]},
$$

If $\xi_{i}$ is substituted by $x_{i}$ via

$$
x_{i}=\frac{\xi_{i}-\mu}{\sigma},
$$

where $(i=1,2)$, we get, after expanding the cosh-function and some rearranging (Appendix B),

$$
\mathcal{L}_{d, s}(\mathbf{x})=\frac{e^{d^{\prime} x_{1}}+e^{d^{\prime} x_{2}}}{1+e^{d^{\prime}\left(x_{1}+x_{2}\right)}}
$$

This expression is identical to the likelihood ratio from Noreen's (1981) Rule 6. Assuming that there is no bias, we have $C=1$. Solving Equation 10 for $\mathcal{L}_{d s}(\mathbf{x})>1$ yields (Appendix B)

$$
x_{1} x_{2}<0,
$$

which is in fact Noreen's (1981) decision Rule 6'. The region defined by Equation 11 is the same as that described by Macmillan and Creelman (1991, p. 144) for the case in which the observations are independent. Equation 11 states that the observer should respond "different" if the observations are in the second or fourth quadrant of the $\left\langle x_{1}, x_{2}\right\rangle$ plane, and "same" otherwise. Written in terms of $\xi$, Equation 11 becomes

$$
\left(\xi_{1}-\mu\right)\left(\xi_{2}-\mu\right)<0
$$


which is the decision rule given in the introduction. It shows that the observer needs to know $\mu$ to make the optimal decision.

The optimum decision rule for $C \neq 1$ is obtained by solving Equation 10 for $\mathcal{L}_{d, s}(\mathbf{x})>C$. This will be done below. For $C>1$ the observer does best to respond "different" if the observations $x_{1}$ and $x_{2}$ are in the shaded areas of the upper panel in Figure 1, and "same" otherwise. For $C<1$, the observer should respond "different" if the observation pair is in the shaded area of the lower panel in Figure 1 . The expected values of the four density functions are now (due to the transformation of Equation 9) $\left[\frac{d^{\prime}}{2} \frac{d^{\prime}}{2}\right]^{T}$ for $\left\langle S_{B}, S_{B}\right\rangle,\left[-\frac{d^{\prime}}{2} \frac{d^{\prime}}{2}\right]^{T}$ for $\left\langle S_{A}, S_{B}\right\rangle,\left[-\frac{d^{\prime}}{2}-\frac{d^{\prime}}{2}\right]^{T}$ for $\left\langle S_{A}, S_{A}\right\rangle$, and $\left[\frac{d^{\prime}}{2}-\frac{d^{\prime}}{2}\right]^{T}$ for $\left\langle S_{B}, S_{A}\right\rangle$.

\section{The Optimum Decision Rule for Highly Correlated Observations}

When $\sigma_{R}^{2} \gg \sigma^{2}\left(\varepsilon^{2} \longrightarrow 0, \rho \longrightarrow 1\right)$, the observations are highly correlated, and Equation 5 reduces to

$$
\mathcal{L}_{d, s}(\xi)=e^{-\left(d^{\prime} / 2\right)^{2}} \cosh \left[\frac{d^{\prime}}{2} \frac{\xi_{2}-\xi_{1}}{\sigma}\right] .
$$

Note that, for the particular case of highly correlated observations, $\mu$ does not appear in Equation 13. The likelihood ratio is determined by only the difference of the two observations, not their absolute values. Based on Equation 13 , the inequality $\mathcal{L}_{d, s}(\boldsymbol{\xi})>C$ can be expressed as

$$
\left|\xi_{2}-\xi_{1}\right|>\frac{2 \sigma}{d^{\prime}} \cosh ^{-1}\left[C e^{\left(d^{\prime} / 2\right)^{2}}\right]
$$

where $\cosh ^{-1}$ is the inverse function of the hyperbolic cosine function. For given values of $C, \sigma$, and $d^{\prime}$, the quantity on the right-hand side of Equation 14 is a constant. This decision rule - which was already mentioned in the introduction -is identical to the rule suggested by Sorkin (1962). By taking the absolute value of the difference between the two observations obtained on any one trial, the decision rule cancels any common variability-that is, positive correlations-associated with the observations. By making the transformation of Equation 9, we obtain

$$
\left|x_{2}-x_{1}\right|>\frac{2}{d^{\prime}} \cosh ^{-1}\left[C e^{\left(d^{\prime} / 2\right)^{2}}\right] .
$$

The values for the pair $\left(x_{1}, x_{2}\right)$ that satisfy Equation 15 are indicated by the shaded region of Figure 2 . Thus, for the highly correlated case, the observer does best to respond "different" if Equation 15 is true.

\section{CALCULATING THE PROBABILITIES OF CORRECT AND INCORRECT "DIFFERENT" RESPONSES (ROC)}

If a correct or an incorrect "different" response in a samedifferent task is equated with a "hit" or a "false alarm" in a yes-no task, then an ROC function can be obtained for the same-different task, which shows how $P($ " $D$ " $\mid d)$ varies with $P$ (" $D$ " $\mid s)$, where $d$ and $s$ stand for trials that contain two different or two same stimuli, respectively.
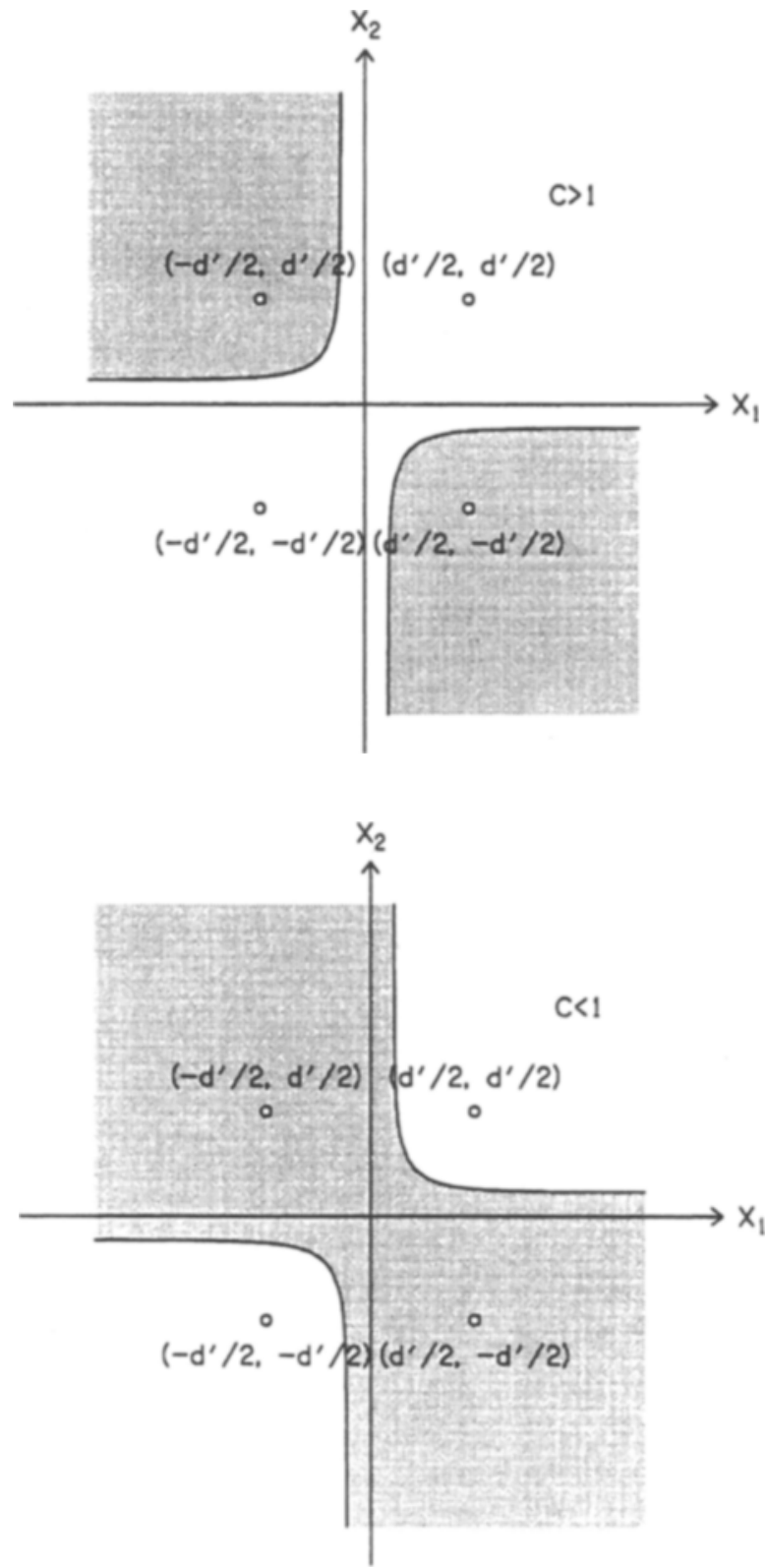

Figure 1. Decision space for independent observations $\left(\sigma_{R}^{2}=0\right)$. The four probability density functions $\langle B B\rangle,\langle A B\rangle,\langle A A\rangle$, and $\langle B A\rangle$ associated with either the same or the different hypothesis are centered at $\left(d^{\prime} / \mathbf{2}, d^{\prime} / 2\right),\left(-d^{\prime} / \mathbf{2}, d^{\prime} / 2\right),\left(-d^{\prime} / 2,-d^{\prime} / 2\right)$, and $\left(d^{\prime} / 2,-d^{\prime} / 2\right)$, respectively. The shaded areas indicate where $\mathcal{L}_{d, s}(x)>C$ is true; see Equation 10 for the expression of $\mathcal{L}_{d, s}(\mathbf{x})$. The boundaries of the shaded area are given by Equation 16. The upper panel is for $C>1$, and the lower panel is for $C<1$. The response bias is altered by changing the value of the criterion $C$.

\section{The ROC Curves for Independent Observations}

Figure 1 shows the $\left\langle x_{1}, x_{2}\right\rangle$ decision space for the samedifferent task where observations are independent $\left(\sigma_{R}^{2}=0\right)$. The upper panel is for $C>1$, and the lower panel for $C<1$. The shaded areas indicate the acceptance region for "different" responses, where $\mathcal{L}_{d s}(\mathbf{x})>C$ (given by Equation 10) is true. The boundaries of the acceptance regions are given by (Appendix B) 


$$
x_{2}=v\left(x_{1}\right)=\frac{1}{d^{\prime}} \ln \left[\frac{C-e^{d^{\prime} x_{1}}}{1-C e^{d^{\prime} x_{1}}}\right] .
$$

By integrating the probability density functions over the shaded regions, we obtain the probabilities of correct and incorrect "different" responses (Appendix B):

$$
\begin{aligned}
P\left(" D^{\prime} \mid d\right)= & \int_{u}^{\infty} \Phi\left(v\left(x_{1}\right)-\frac{d^{\prime}}{2}\right) \phi\left(x_{1}+\frac{d^{\prime}}{2}\right) d x_{1} \\
& +\int_{u}^{\infty} \Phi\left(v\left(x_{1}\right)+\frac{d^{\prime}}{2}\right) \phi\left(x_{1}-\frac{d^{\prime}}{2}\right) d x_{1},
\end{aligned}
$$

and

$$
P(“ D ” \mid s)=2 \int_{u}^{\infty} \Phi\left(v\left(x_{1}\right)-\frac{d^{\prime}}{2}\right) \phi\left(x_{1}-\frac{d^{\prime}}{2}\right) d x_{1},
$$

where $\phi(z)$ and $\Phi(z)$ are the Gaussian and the cumulative Gaussian probability density function, respectively, and $u=\frac{1}{d} \ln (C)$. For $C<1$, the two probabilities can be derived from those for $C>1$ on the basis of Equations 17 and 18, by using the property that

$$
P_{\mathcal{L}=C}(\text { " } D " \mid d)=1-P_{\mathcal{L}=1 / C}(\text { " } D " \mid s)
$$

and

$$
P_{L=C}(“ D " \mid s)=1-P_{\mathcal{L}=1 / C}(“ D " \mid d) \text {. }
$$

Figure 3 shows the ROC curves generated via Equations 17-20. The probability of a correct "different" response, $P($ " $D$ " $\mid d)$, is plotted as a function of the probability of an incorrect "different" response, $P($ " $D$ " $\mid s)$. The parameter is the value of $d^{\prime}$. Each ROC curve is symmetric about the

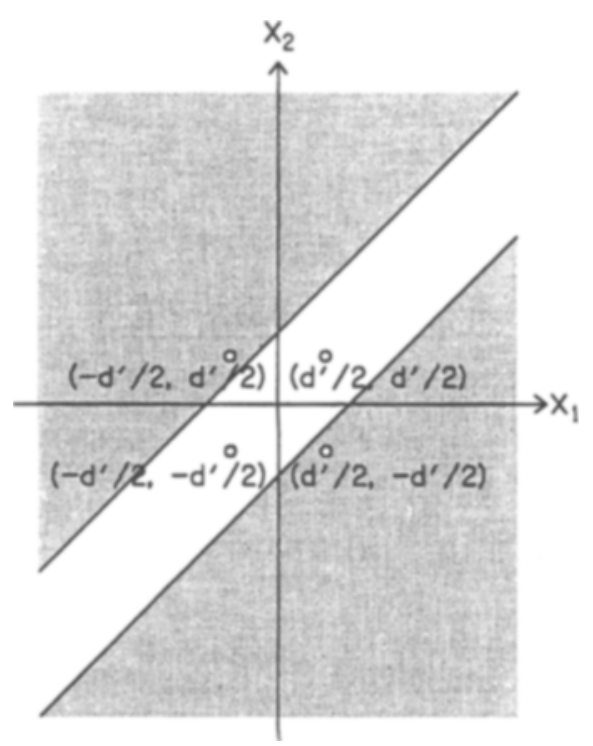

Figure 2. Decision space for highly correlated observations $\left(\sigma_{R}^{2} \gg \sigma^{2}\right)$. The four probability density functions $\langle B \boldsymbol{B}\rangle,\langle\boldsymbol{A B}\rangle$, $\langle A A\rangle$, and $\langle B A\rangle$ associated with either the same or the different hypothesis are centered at $\left(d^{\prime} / 2, d^{\prime} / 2\right),\left(-d^{\prime} / 2, d^{\prime} / 2\right),\left(-d^{\prime} / 2,-d^{\prime} / 2\right)$, and $\left(d^{\prime} / 2,-d^{\prime} / 2\right)$, respectively. The acceptance region for the "different" response is indicated by the shaded areas, where Equation 15 is true. When the criterion $C$ is altered, the unshaded area changes in width.

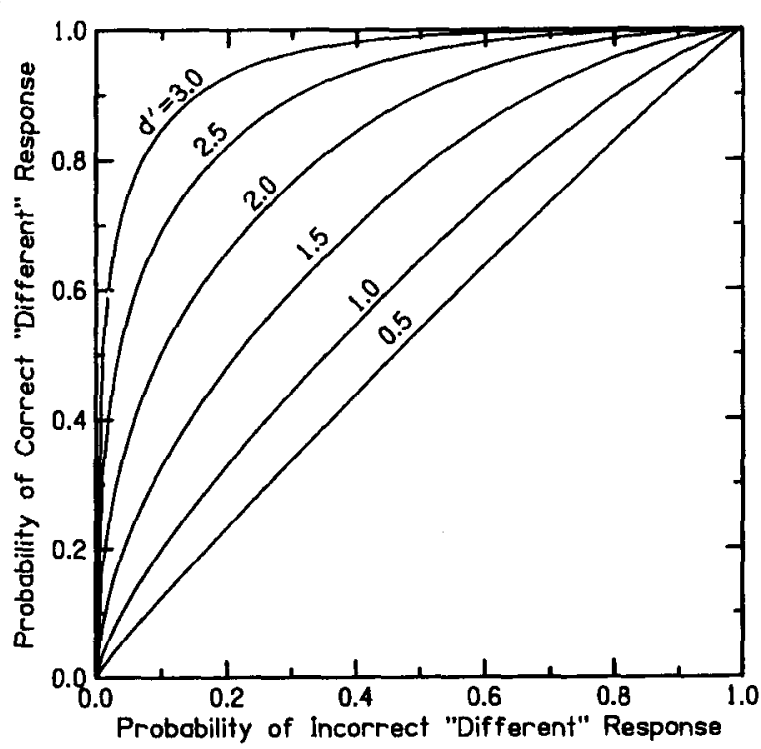

Figure 3. Receiver-operating characteristic (ROC) curves for independent observations $\left(\sigma_{R}^{2}=0\right)$ using the following decision rule: Respond "different" iff $\mathcal{L}_{d, s}(x)>C$. The expression of the likelihood ratio, $\mathcal{L}_{d, s}(x)$, is given by Equation 10. The parameter is the $d^{\prime}$ value. The curves are calculated by using Equations 17-20.

minor diagonal. Macmillan and Creelman (1991, p. 145) suggested an approximation method for calculating the $d$ ' values on the basis of $P($ " $D$ " $\mid d)$ and $P(" D$ " $\mid s)$ values. Except for extreme values of the criterion (i.e., for $C \ll 1$ and $C \gg 1$ ), the approximation is reasonably accurate; their approximated $d^{\prime}$ values and our calculated $d^{\prime}$ values are within a few percentage points.

\section{The ROC Curves for Highly Correlated Observations}

For highly correlated observations, the decision space is shown in Figure 2. One could follow the previous section and calculate the probabilities $P($ " $D$ " $\mid d)$ and $P($ " $D$ " $\mid s)$ by integrating the joint density functions over the shaded regions; however, these probabilities can be obtained via a simpler approach, described by Sorkin (1962). One can express the observations on a single trial as $\xi_{1}=\zeta_{1}+\omega_{r}$ and $\xi_{2}=\zeta_{2}+\omega_{r}$, where $\zeta_{1}$ and $\zeta_{2}$ are independent of each other, both having a variance of $\sigma^{2}$, and the common term $\omega_{r}$ has zero mean and variance $\sigma_{R}^{2}$. Because the optimum decision statistic is the absolute value of the difference between the two observations, we shall work with the difference of the two observations, normalized with respect to $\sigma, z=\left(\xi_{2}-\xi_{1}\right)$ $\sigma=\left(\zeta_{2}-\zeta_{1}\right) / \sigma$. Note that the common noise $\omega_{r}$ is canceled in $z$. Based on this relationship, the calculation of the ROC functions requires only one-dimensional integrations.

For the same hypothesis, the probability density function of the difference $z$ is a Gaussian having a mean of zero and a variance of 2 (the sum of the two independent variances). For the different hypothesis, the density function is a composite function of two parts. One is a Gaussian having a mean of $\left(\mu_{B}-\mu_{A}\right) / \sigma=d^{\prime}$ and a variance of 2 , with the 
other having a mean of $-\left(\mu_{B}-\mu_{A}\right) / \sigma=-d^{\prime}$ and a variance of 2. Figure 4 shows the decision space for the present task. For a given criterion $C$, the observer responds "different" iff the difference score $z$ falls into the shaded area of Figure 4. $P($ " $D$ " $\mid d)$ is the integration of the two different density functions over the shaded areas. Likewise, $P($ " $D$ " $\mid s)$ is obtained by an integration of the same density function over the shaded areas. The two probabilities are presented here in a form similar to that given by Sorkin (1962):

$$
\begin{aligned}
P(“ D ” \mid d) & =1-\frac{1}{2} \int_{-z_{C}}^{z_{C}}\left[\phi\left(\frac{z-d^{\prime}}{\sqrt{2}}\right)+\phi\left(\frac{z+d^{\prime}}{\sqrt{2}}\right)\right] d z \\
& =2-\Phi\left(\frac{z_{C}-d^{\prime}}{\sqrt{2}}\right)-\Phi\left(\frac{z_{C}+d^{\prime}}{\sqrt{2}}\right)
\end{aligned}
$$

and

$$
\begin{aligned}
P\left(“ D^{\prime \prime} \mid s\right) & =1-\int_{-z_{C}}^{z_{C}} \phi\left(\frac{z}{\sqrt{2}}\right) d z \\
& =2-2 \Phi\left(\frac{z_{C}}{\sqrt{2}}\right)
\end{aligned}
$$

where $z_{C}$ is obtained from Equation 14

$$
\mathrm{z}_{C}=\frac{2}{d^{\prime}} \cosh ^{-1}\left[C e^{\left(d^{\prime} / 2\right)^{2}}\right] .
$$

Figure 5 shows the ROC curves generated from Equations 22 and 24 . The parameter is $d^{\prime}$. The ROC curves are slightly asymmetric about the minor diagonal. Each curve tends to be farther apart from the major diagonal near the origin $(0,0)$ than near $(1,1)$. Our calculations confirm Macmillan and Creelman's (1991).

\section{HOW TO DETERMINE THE DECISION RULE USED BY THE OBSERVER}

For a given $d^{\prime}$, the ROC curves generated from the two decision statistics (Equation 8 for independent, and Equation 13 for highly correlated observations) are clearly different. Suppose, for example, that an experiment produced values of $P(" D$ " $\mid d)=0.8$ and $P(" D " \mid s)=0.2$. Then one would estimate that $d^{\prime}=2.4$, given the independentobservation decision rule (Figure 3 ), and that $d^{\prime}=3.0$, given the second decision rule (Figure 5), a difference in $d^{\prime}$ of about $20 \%$. Such differences in $d^{\prime}$ suggest that it would be desirable to have a means of determining which of the two decision statistics the observer actually uses.

We now present a procedure that may allow the experimenter to distinguish between the two potential decision rules. In our application, we will use a modification of Berg's (1989) conditional-on-a-single-stimulus (COSS) technique as suggested by Richards and Zhu (1994) and by Lutfi (1995). (Despite the modification, we will still refer to it as the COSS procedure.) The COSS procedure calculates a correlation between the observer's responses and alterations of the stimuli imposed by the experimenter on each trial, which we call perturbations. We have not attempted to use the technique experimentally, but the following computer simulations suggest that the technique may be of value. In the example, we consider only the two extreme decision rules (Equations 8 and 13). Human ob-

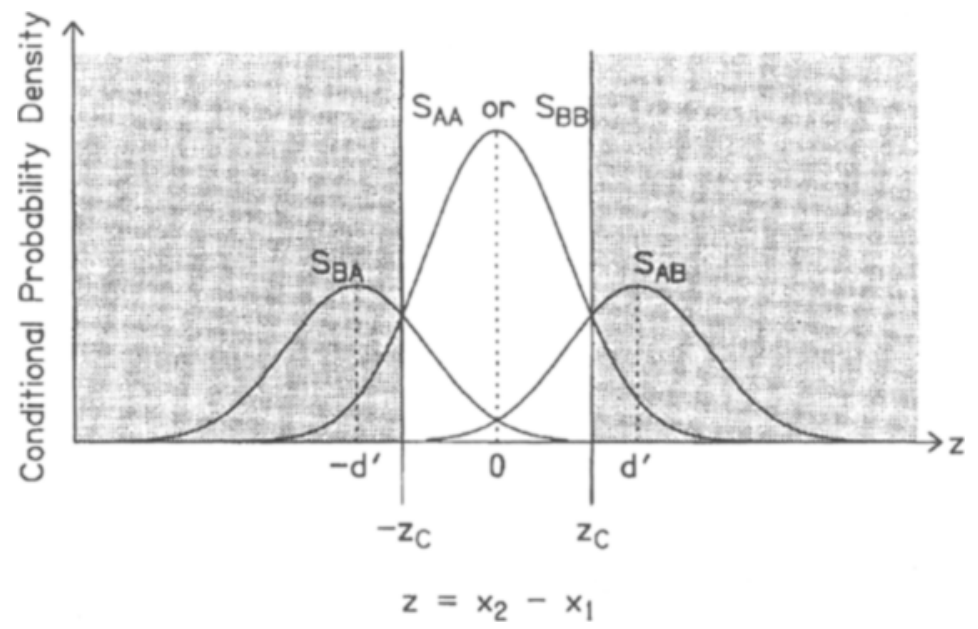

Figure 4. Simplified conditional probability density functions for the different and same hypotheses and decision space for the same-different task where the observations are highly correlated. The abscissa $(z)$ is the difference between the observation of the second interval and the first interval, normalized with respect to $\sigma$. The middle function denotes the Gaussian probability density function given that a stimulus pair $A \boldsymbol{A}$ or $B B$ was presented. Its variance equals 2 , and the area under the curve equals unity. The two other distributions denote the probability density functions given a pair $A B$ or $B A$, respectively. For both distributions the variance equals 2 , but the area under each curve equals 0.5 . The observer responds "different" iff the $z$ value falls into the shaded areas. The boundaries of the shaded areas, $-z_{C}$ and $z_{C}$, correspond to the decision criterion. 


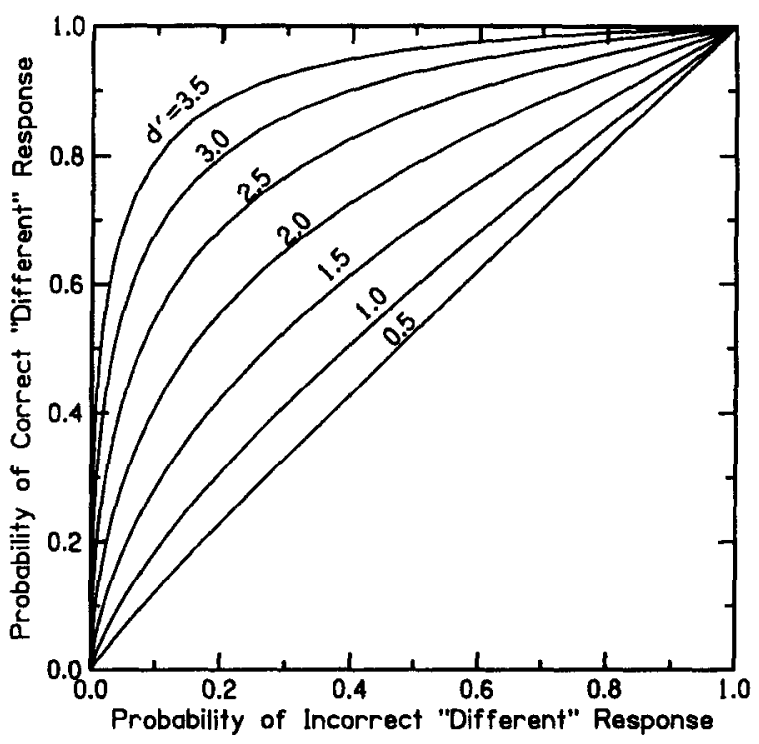

Figure 5. Receiver-operating characteristic (ROC) curves for highly correlated observations using the following decision rule: Respond "different" iff Equation 15 is true. The parameter is the $d^{\prime}$ value. The curves are calculated by using Equations 22 and 24.

servers may, however, adopt more complicated rules or may even blend the two extreme rules. For a more detailed discussion of the COSS technique and its limitations, the original papers should be consulted.

On each trial, the experimenter modifies each stimulus of the same-different pair by adding a random and independent perturbation to it. For example, if the problem were to discriminate whether two tones had the same or different frequencies, we might modify the frequency of each member of the tone pair by changing it by an amount $\delta f_{1}$ or $\delta f_{2}$, respectively. The perturbation value is a random variable drawn from a Gaussian distribution with zero mean and variance $\sigma_{p}^{2}$. Thus, contrary to the roving standard, the present perturbation is different for each stimulus interval. Note that if the observer were infinitely sensitive, even the same trials would appear to be different, because it is unlikely that exactly the same perturbation would be applied to both stimuli of a same pair. This is probably not a problem, because the perturbation can be chosen to be small yet still useful. In other applications, it has been found that the perturbation is largely unnoticed by the listener (Berg \& Green, 1990). Next, we record for each trial the values of the two perturbations and the observer's response $R$ (e.g., $R=1$ for a "different," and $R=0$ for a "same," response).

At the conclusion of a set of trials, we compute two correlation coefficients: one between the binary response given on that trial and the perturbation of the first stimulus, and a second one based on that same response and the perturbation of the second stimulus of the pair. There are four types of trials, $\left\langle S_{A}, S_{A}\right\rangle,\left\langle S_{A}, S_{B}\right\rangle,\left\langle S_{B}, S_{A}\right\rangle$, and $\left\langle S_{B}, S_{B}\right\rangle$, so that a total of eight correlations can be computed. For each trial type, the correlation between the response and the perturbation of the second interval can be plotted as a function of the correlation between the response and the perturbation of the first interval. This has been done in Figure 6 . Using a computer to simulate performance in the samedifferent task, we find that the pattern of correlations is quite different for the two different decision rules. In each of the four panels, representing the different types of trials, the crosses are the correlations obtained by using the optimum decision rule for independent observations (Equation $8, C=1$ ), and the circles are the correlations obtained by using the optimum decision rule for highly correlated observations (Equation 13, C=1). Each correlation value (circle or cross) is based on 100 trials. The simulation was repeated 2,000 times in order to give us the cluster of points. As can be seen in Figure 6, for each trial type, the two sets of correlation values cluster in different regions depending on the rule used by the decision maker. For example, in the lower left panel, for $\left\langle S_{A}, S_{A}\right\rangle$, if a point falls above the line, it is more likely to be generated from the optimum decision rule for correlated observations; if it falls below the line, the other decision rule is more likely. The lines are simple linear functions that were empirically adjusted to separate the two clusters. The error rate is $1 \%$ for the same stimulus pairs (lower left and upper right panels of Figure 6) and about $9 \%$ for the different stimulus pairs.

An experimenter can locate the point on the appropriate panel of Figure 6 by segregating the trial types and computing the two correlations between the observer's responses and the perturbations applied to the first and second stimuli. With only one trial type, the error rate classifying the decision rule used by the observer can be less than $1 \%$. Lower error rates can be obtained if all four trial types are used.

We used specific parameter values when conducting the computer simulations. The variance of the internal noise of the observer was set to $\sigma^{2}$; that of the perturbation $\sigma_{p}^{2}$ was given a value $2.25 \sigma^{2}$, to give a total variance of $\sigma_{T}^{2}=\sigma^{2}+\sigma_{p}^{2}=3.25 \sigma^{2}$. The means $\mu_{A}$ and $\mu_{B}$ of the two probability density functions were adjusted to produce a normalized difference of $\left(\mu_{B}-\mu_{A}\right) / \sigma=1.8$.

To apply this technique to human observers, the experimenter must adjust two experimental variables in order to obtain useful correlation coefficients. They are the value of the perturbation, $\sigma_{p}$, and the difference between the means of the two distributions, $\mu_{B}-\mu_{A}$. If the perturbation is too small, it will not influence the observer's responses. If the perturbation is too large, all trials will appear to be "different." To determine the most useful perturbation value, the experimenter can estimate the internal noise by measuring the subject's threshold $d^{\prime}=\left(\mu_{B}-\mu_{A}\right) / \sigma$ without perturbations: Call the resulting threshold $\Delta_{t h}$ for $d^{\prime}=1$, which we can use as an estimate of the listener's internal noise, $\sigma$. We recommend that the size of the perturbation be about $1.5 \Delta_{t h}$, as it was in the simulation, so that the subject's response is influenced more by perturbation than by internal noise. The size of the signal must also be selected with care. It should not be too large; otherwise, the responses 


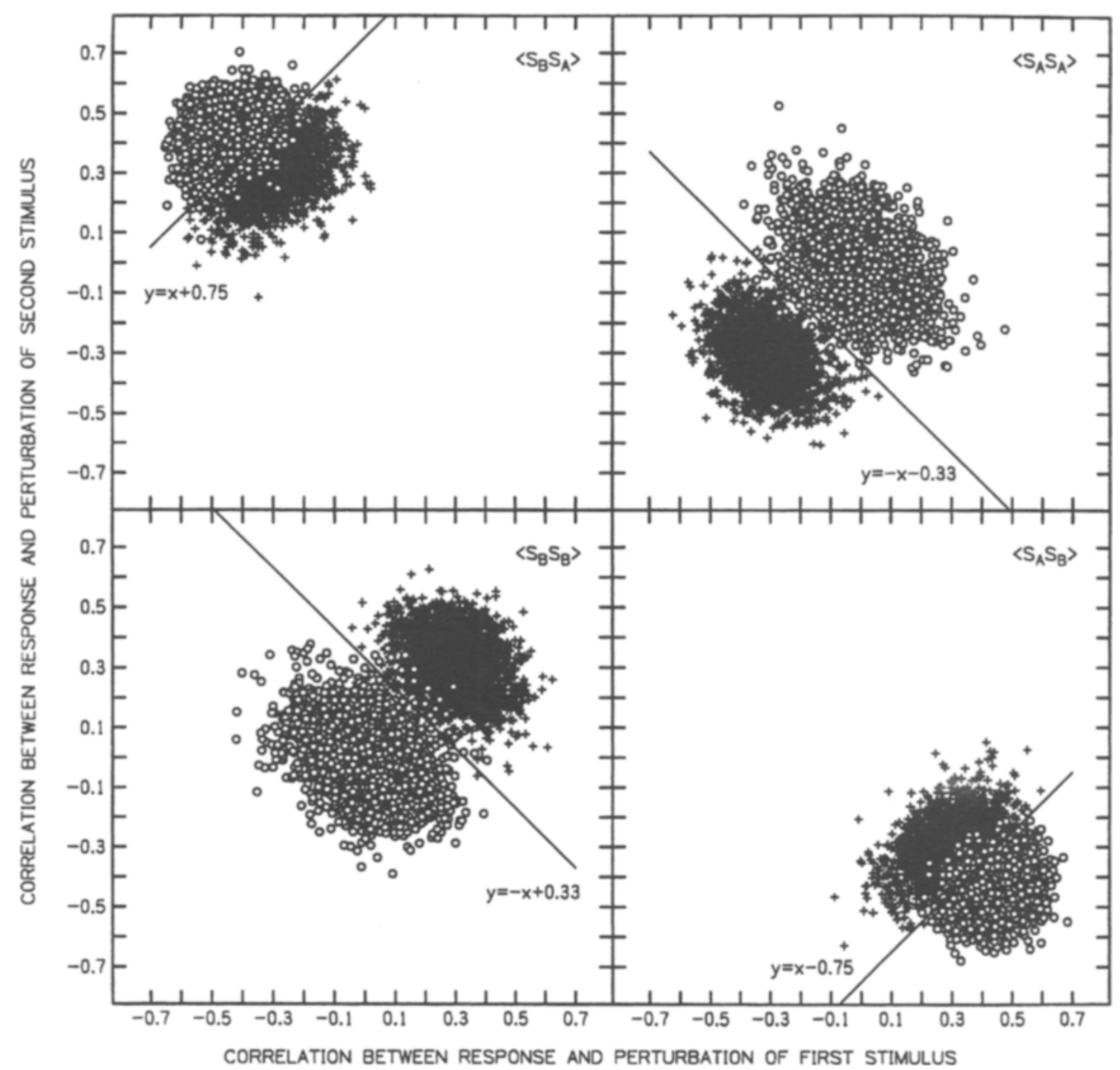

Figure 6. Computer simulation results of the correlation coefficients between the observer's responses and perturbations imposed on the observations. The observations on each trial are assumed to be independent. The total variance is set to be $3.25 \sigma^{2}$, which includes a variance of $\sigma^{2}$ for the internal noise and a variance $\sigma_{p}^{2}=2.25 \sigma^{2}$ for the perturbation. Each panel is for a particular type of stimulus pairs. The ordinate is the correlation coefficient between the responses ( 1 for "different," and 0 for "same") and the perturbations imposed to the second stimulus; the abscissa is the correlation coefficient between the responses and the perturbations imposed to the first stimulus. Each panel has 2,000 crosses and circles, and each point is based on 100 trials. The crosses are generated with the optimum decision statistic for independent observations (Equation 11), and the circles with the optimum decision statistic for highly correlated observations (Equation 15 with $C=1$ ). Each straight line (with equation) represents an empirically determined criterion for each type of trial. The lines were chosen so that minimum numbers of crosses and circles went across them. In the lower left panel $\left(\left\langle S_{A}, S_{A}\right\rangle\right)$, for example, if an experimenter obtains a point that falls below the line, the point is more likely to be generated from the optimum decision rule for independent observations; if it falls below the line, the other decision rule is more likely. With this procedure, the error rate is about $1 \%$ for each of the two same stimulus pairs (the lower left and upper right panels) and about $9 \%$ for each of the two different stimulus pairs.

will all be correct. Nor should it be too small; otherwise, the subject may not maintain a reasonable decision rule in the task.

\section{SUMMARY}

In this paper, we have derived the optimum (likelihoodratio) decision statistic for a same-different task, assuming that the observations are partially correlated. Under two special cases, the optimum decision statistic is identical to two previously suggested decision statistics. When the correlation is zero-so the observations are independent - the optimum decision rule is identical to the rule suggested by Noreen (1981, cf. Macmillan \& Creelman, 1991). When the observations are highly correlated, the optimum decision rule is identical to the rule suggested by Sorkin (1962). Using the decision statistic optimal for either independent or highly correlated observations, we 
calculated for given $d^{\prime}$ values the probability of a correct "different" response as a function of the probability of an incorrect "different" response (ROC). A correlation analysis based on the COSS procedure provides a means for empirically distinguishing the actual decision rule used by the observer.

\section{REFERENCES}

BERG, B. G. (1989). Analysis of weights in multiple observation tasks. Journal of the Acoustical Society of America, 86, 1743-1746.

BERG, B. G., \& GREEN, D. M. (1990). Spectral weights in profile listening. Journal of the Acoustical Saciety of America, 88, 758-766.

LUTFI, R. A. (1995). Correlation coefficients and correlation ratios as estimates of observer weights in multiple-observation tasks. Journal of the Acoustical Society of America, 97, 1333-1334.

MaCMillan, N. A., \& Creelman, C. D. (1991), Detection theory: A user's guide. Cambridge: Cambridge University Press.

NoREEN, D. L. (1981). Optimal decision rules for some common psychophysical paradigms. In S. Grossberg (Ed.), Mathematical psychology and psychophysiology (Proceedings of the Symposium in Applied Mathematics of the American Mathematical Society and the Society for Industrial and Applied Mathematics) (SIAM-AMS Proceedings, Vol. 13, pp. 237-279). Providence, RI: American Mathematical Society.

RICHARDS, V. M., \& ZHU, S. (1994). Relative estimates of combination weights, decision criteria, and internal noise based on correlation coefficients. Journal of the Acoustical Society of America, 95, 423-434.

SoRKIN, R. D. (1962). Extension of the theory of signal detectability to matching paradigms in psychoacoustics. Journal of the Acoustical Society of America, 34, 1745-1751.

Versfeld, N. J., DAI, H., \& GreEn, D. M. (1996). The optimum decision rules for the oddity task. Perception \& Psychophysics, 58, 10-21.

\section{APPENDIX A}

The Optimum Decision Rule for a Same-Different Task in the Presence of Correlation Between Observations

The pair of observations $\xi_{1}$ and $\xi_{2}$, written as a vector $\xi=$ $\left[\xi_{1} \xi_{2}\right]^{T}$, obtained in a same-different task, has equal probability to be drawn from four joint Gaussian distributions having means of $\boldsymbol{\mu}_{A A}=\left[\mu_{A} \mu_{A}\right]^{T}, \boldsymbol{\mu}_{A B}=\left[\mu_{A} \mu_{B}\right]^{T}, \boldsymbol{\mu}_{B A}=\left[\mu_{B} \mu_{A}\right]^{T}$, and $\boldsymbol{\mu}_{B B}=$ $\left[\mu_{B} \mu_{B}\right]^{T}$. Here, $T$ stands for transpose. Under the assumption that the two observations, $\xi_{1}$ and $\xi_{2}$, are partially correlated, the density functions are

$$
p d f_{X X}(\boldsymbol{\xi})=\frac{1}{2 \pi \sqrt{\left|\Sigma^{2}\right|}} e^{-\frac{1}{2}\left(\boldsymbol{\xi}-\boldsymbol{\mu}_{X X}\right)^{T} \Sigma^{-2}\left(\boldsymbol{\xi}-\boldsymbol{\mu}_{X X}\right)}
$$

where $X X=A A, A B, B A$, or $B B$, and where $\left|\Sigma^{2}\right|$ is the determinant of the covariance matrix $\Sigma^{2}$

$$
\Sigma^{2}=\left[\begin{array}{lr}
\sigma^{2}+\sigma_{R}^{2} & \sigma_{R}^{2} \\
\sigma_{R}^{2} & \sigma^{2}+\sigma_{R}^{2}
\end{array}\right],
$$

in which $\sigma^{2}$ represents the independent variance of each observation and $\sigma_{R}^{2}$ represents the common variance between the two observations. The inverse of the covariance matrix is

$$
\begin{aligned}
\Sigma^{-2} & =\frac{1}{\sigma^{2}\left(\sigma^{2}+2 \sigma_{R}^{2}\right)}\left[\begin{array}{lr}
\sigma^{2}+\sigma_{R}^{2} & -\sigma_{R}^{2} \\
-\sigma_{R}^{2} & \sigma^{2}+\sigma_{R}^{2}
\end{array}\right] \\
& =\frac{1}{2 \sigma^{2}}\left[\begin{array}{ll}
\varepsilon^{2}+1 & \varepsilon^{2}-1 \\
\varepsilon^{2}-1 & \varepsilon^{2}+1
\end{array}\right],
\end{aligned}
$$

where we defined for convenience

$$
\varepsilon^{2}=\frac{\sigma^{2}}{\sigma^{2}+2 \sigma_{R}^{2}} .
$$

We have introduced $\varepsilon^{2}$ rather than $\varepsilon$ for no other reason than to indicate that its value is always positive. The likelihood ratio of being "different" over "same" is

$$
\mathcal{L}_{d, s}(\boldsymbol{\xi})=\frac{p d f_{d}(\boldsymbol{\xi})}{p d f_{s}(\boldsymbol{\xi})}=\frac{\frac{1}{2}\left[p d f_{A B}(\boldsymbol{\xi})+p d f_{B A}(\boldsymbol{\xi})\right]}{\frac{1}{2}\left[p d f_{A A}(\boldsymbol{\xi})+p d f_{B B}(\boldsymbol{\xi})\right]} .
$$

The exponential part of each density function, $p d f_{X X}(\boldsymbol{\xi})$, is

$$
-\frac{1}{2}\left[\boldsymbol{\xi}^{T} \Sigma^{-2} \boldsymbol{\xi}-2 \boldsymbol{\xi}^{T} \Sigma^{-2} \boldsymbol{\mu}_{X X}+\boldsymbol{\mu}_{X X}^{T} \Sigma^{-2} \boldsymbol{\mu}_{X X}\right] .
$$

Because $\xi^{T} \Sigma^{-2} \xi$ is common to all four density functions, it is canceled in the expression of $\mathcal{L}_{d, 5}(\xi)$. Thus, we only need to consider the linear term of $\boldsymbol{\xi}$ and the constant term.

Insertion of the expressions of the density functions from Equation $\mathrm{A} 1$ into Equation $\mathrm{A} 5$ yields, with the aid of Equation $\mathrm{A} 6$,

$$
\mathcal{L}_{d, s}(\boldsymbol{\xi})=\frac{e^{K_{A B}+e^{K_{B A}}}}{e^{K_{A A}+e^{K_{B B}}}}
$$

where

$$
K_{X X}=\left[\boldsymbol{\xi}^{T} \Sigma^{-2} \boldsymbol{\mu}_{X X}-\frac{1}{2} \boldsymbol{\mu}_{X X}^{T} \Sigma^{-2} \boldsymbol{\mu}_{X X}\right] .
$$

By using the expressions for $\boldsymbol{\xi}, \boldsymbol{\mu}_{X X}$, and $\boldsymbol{\Sigma}^{-2}$, but also making use of the definitions of $\mu=\left(\mu_{A}+\mu_{B}\right) / 2, \varepsilon^{2}=\sigma^{2} /\left(\sigma^{2}+2 \sigma_{R}^{2}\right)$, and $d^{\prime}=\left(\mu_{B}-\mu_{A}\right) / \sigma$, we obtain

$$
\begin{gathered}
K_{A A}=\varepsilon^{2}\left[\left(\frac{\mu}{\sigma}-\frac{d^{\prime}}{2}\right)\left(\frac{\xi_{1}+\xi_{2}}{\sigma}\right)-\left(\frac{\mu}{\sigma}-\frac{d^{\prime}}{2}\right)^{2}\right], \\
K_{B B}=\varepsilon^{2}\left[\left(\frac{\mu}{\sigma}+\frac{d^{\prime}}{2}\right)\left(\frac{\xi_{1}+\xi_{2}}{\sigma}\right)-\left(\frac{\mu}{\sigma}+\frac{d^{\prime}}{2}\right)^{2}\right], \\
K_{A B}=-\left(\frac{d^{\prime}}{2}\right)^{2}-\varepsilon^{2}\left(\frac{\mu}{\sigma}\right)^{2}+\varepsilon^{2} \frac{\mu}{\sigma} \frac{\xi_{1}+\xi_{2}}{\sigma}+\frac{d^{\prime}}{2} \frac{\xi_{2}-\xi_{1}}{\sigma},
\end{gathered}
$$

and

$$
K_{B A}=-\left(\frac{d^{\prime}}{2}\right)^{2}-\varepsilon^{2}\left(\frac{\mu}{\sigma}\right)^{2}+\varepsilon^{2} \frac{\mu}{\sigma} \frac{\xi_{1}+\xi_{2}}{\sigma}-\frac{d^{\prime}}{2} \frac{\xi_{2}-\xi_{1}}{\sigma} .
$$

Substituting the resulting expressions for all four density functions into Equation A7, and simplifying, we obtain

$$
\mathcal{L}_{d, s}(\boldsymbol{\xi})=\exp \left[-\left(\frac{d^{\prime}}{2}\right)^{2}\left(1-\varepsilon^{2}\right)\right] \frac{\cosh \left[\frac{d^{\prime}}{2} \frac{\xi_{2}-\xi_{1}}{\sigma}\right]}{\cosh \left[\frac{d^{\prime}}{2} \varepsilon^{2} \frac{\xi_{1}+\xi_{2}-2 \mu}{\sigma}\right]},
$$

where the hyperbolic cosine function is defined as

$$
\cosh (z)=\frac{e^{-z}+e^{z}}{2} .
$$

This expression is identical to Equation 5 in the main text.

\section{APPENDIX B \\ Calculation of the Receiver-Operating Characteristic (ROC) Functions for Independent Observations}

When the observations are independent, $\sigma_{R}^{2}=0$, thus $\varepsilon^{2}=1$, and Equation A13 (Equation 5 in the main text) reduces to 


$$
\mathcal{L}_{d, s}(\boldsymbol{\xi})=\frac{\cosh \left[\frac{d^{\prime}}{2} \frac{\xi_{2}-\xi_{1}}{\sigma}\right]}{\cosh \left[\frac{d^{\prime}}{2} \frac{\xi_{1}+\xi_{2}-2 \mu}{\sigma}\right]} .
$$

For convenience, we make the transformation

$$
x_{i}=\frac{\xi_{i}-\mu}{\sigma},
$$

where $(i=1,2)$, which changes Equation B1 into

$$
\mathcal{L}_{d, s}(\boldsymbol{\xi})=\frac{\cosh \left[\frac{d^{\prime}}{2}\left(x_{2}-x_{1}\right)\right]}{\cosh \left[\frac{d^{\prime}}{2}\left(x_{1}+x_{2}\right)\right]} .
$$

Expanding the cosh functions, by use of the definition given in Equation A14, we obtain, after multiplication of numerator and denominator by a factor of $\exp \left[\frac{d^{\prime}}{2}\left(x_{1}+x_{2}\right)\right]$ and simplification,

$$
\mathcal{L}_{d, s}(\xi)=\frac{e^{d^{\prime} x_{1}}+e^{d^{\prime} x_{2}}}{1+e^{d^{\prime}\left(x_{1}+x_{2}\right)}},
$$

which is identical to the likelihood ratio from Noreen's (1981) Rule 6. Solving Equation B4 for $\mathcal{L}_{d, s}(\xi)=C(C \neq 1)$ yields Equation 16 in the main text

$$
x_{2}=v\left(x_{1}\right)=\frac{1}{d^{\prime}} \ln \left[\frac{C-e^{d^{\prime} x_{1}}}{1-C e^{d^{\prime} x_{1}}}\right],
$$

which describes the boundaries in Figure 1. Note that Equation B5 will still be correct when the two variables $x_{1}$ and $x_{2}$ are interchanged. For the special case of $\mathcal{L}_{d . s}(\xi)=1$ (no bias), Equation $\mathrm{B} 4$ reduces to $x_{1} x_{2}=0$, so that the boundaries are defined by the $x_{1}$ - and $x_{2}$-axis. The expected values of the four density functions are now (owing to the transformation of Equation B2) $\left[\frac{d^{\prime}}{2} \frac{d^{\prime}}{2}\right]^{T}$ for $\left\langle S_{B}, S_{B}\right\rangle,\left[-\frac{d^{\prime}}{2} \frac{d^{\prime}}{2}\right]^{T}$ for $\left\langle S_{A}, S_{B}\right\rangle,\left[-\frac{d^{\prime}}{2}-\frac{d^{\prime}}{2}\right]^{T}$ for $\left\langle S_{A}, S_{A}\right\rangle$, and $\left[\frac{d^{\prime}}{2}-\frac{d^{\prime}}{2}\right]^{T}$ for $\left\langle S_{B}, S_{A}\right\rangle^{2}$.

The probabilities of correct and incorrect "different" responses are obtained by integrating the probability density functions over the region of acceptance for the "different" response (the shaded areas in Figure 1). Because here $\sigma_{R}^{2}=0$, the observations are independent; thus the joint probability density functions from Equation $\mathrm{Al}$ can be written as a product of the individual (onedimensional) density functions. The joint density function for $\left\langle S_{A}, S_{B}\right\rangle$ trials, $p d f_{A B}(\mathbf{x})$, can be expressed as

$$
\begin{aligned}
p d f_{A B}(\mathbf{x}) & =\frac{1}{\sqrt{2 \pi}} e^{-\frac{1}{2}\left(x_{1}+\frac{d^{\prime}}{2}\right)^{2}} \cdot \frac{1}{\sqrt{2 \pi}} e^{-\frac{1}{2}\left(x_{2}-\frac{d^{\prime}}{2}\right)^{2}} \\
& =\phi\left(x_{1}+\frac{d^{\prime}}{2}\right) \cdot \phi\left(x_{2}-\frac{d^{\prime}}{2}\right) \\
& =p d f_{A}\left(x_{1}\right) \cdot p d f_{B}\left(x_{2}\right),
\end{aligned}
$$

where $\phi\left(x_{i}\right)$ is the standard Gaussian probability density function having mean zero and unit variance. Likewise, the probability density for $\left\langle S_{B}, S_{A}\right\rangle$ trials is $p d f_{B A}(\mathbf{x})=\phi\left(x_{1}-\frac{d^{\prime}}{2}\right) \cdot \phi\left(x_{2}+\frac{d^{\prime}}{2}\right)$. The composite density function for all different trials is $p d f_{d}(\mathbf{x})=$ $\frac{1}{2}\left[p d f_{A B}(\mathbf{x})+p d f_{B A}(\mathbf{x})\right]$. The probability density for $\left\langle S_{A}, S_{A}\right\rangle$ trials is $p d f_{A A}(\mathbf{x})=\phi\left(x_{1}+\frac{d^{\prime}}{2}\right) \cdot \phi\left(x_{2}+\frac{d^{\prime}}{2}\right)$, and that for $\left\langle S_{B}, S_{B}\right\rangle$ trials is $p d f_{B B}(\mathbf{x})=\phi\left(x_{1}-\frac{d^{\prime}}{2}\right) \cdot \phi\left(x_{2}-\frac{d^{\prime}}{2}\right)$. The composite density function for $p d f_{s}(\mathbf{x})=\frac{1}{2}\left[p d f_{A A}(\mathbf{x})+p d f_{B B}(\mathbf{x})\right]$. Now we derive one part of the ROC curve that is associated with decision criterion values $C>1$ (the upper panel of Figure 1). The rest of the ROC curve, which is associated with $C<1$ (the lower panel of Figure 1), can be derived from the first part based on the property that

$$
P_{\mathcal{L}=C}(“ D ” \mid d)=1-P_{\mathcal{L}=1 / C}(" D " \mid s)
$$

and

$$
P_{L=C}(" D " \mid s)=1-P_{\mathcal{L}=1 / C}(" D " \mid d) \text {. }
$$

The reader may gain some insights about those two relations by comparing the shaded regions of the upper and lower panels of Figure 1 .

For $C>1$ (the upper panel of Figure 1), because the two shaded areas in quadrants II and IV are symmetric with $p d f_{d}(\mathbf{x}), P($ " $D$ "| $d)$ may be obtained by integrating $p d f_{d}(\mathbf{x})$ over quadrant IV and doubling the result. Because $2 p d f_{d}(\mathbf{x})=p d f_{A B}(\mathbf{x})+p d f_{B A}(\mathbf{x})$, we have

$$
\begin{aligned}
P(" D " \mid d)= & \int_{u}^{\infty}\left[\int_{-\infty}^{v\left(x_{1}\right)} \phi\left(x_{1}+\frac{d^{\prime}}{2}\right) \phi\left(x_{1}-\frac{d^{\prime}}{2}\right) d x_{2}\right] d x_{1} \\
& +\int_{u}^{\infty}\left[\int_{-\infty}^{v\left(x_{1}\right)} \phi\left(x_{1}-\frac{d^{\prime}}{2}\right) \phi\left(x_{1}+\frac{d^{\prime}}{2}\right) d x_{2}\right] d x_{1},
\end{aligned}
$$

where $u=\frac{1}{d^{\prime}} \ln (C)$. Therefore, the probability of a correct "different" response, $P($ " $D$ " $\mid d)$, may be expressed as

$$
\begin{aligned}
P(“ D ” \mid d)= & \int_{u}^{\infty} \Phi\left(v\left(x_{1}\right)-\frac{d^{\prime}}{2}\right) \phi\left(x_{1}+\frac{d^{\prime}}{2}\right) d x_{1} \\
& +\int_{u}^{\infty} \Phi\left(v\left(x_{1}\right)+\frac{d^{\prime}}{2}\right) \phi\left(x_{1}-\frac{d^{\prime}}{2}\right) d x_{1},
\end{aligned}
$$

where $\Phi(x)$ is the cumulative Gaussian probability density function.

Likewise, the probability of an incorrect "different" response, $P($ " $D$ " $\mid s)$, given the response criterion, $C$, is the integration of $p d f_{s}(\mathbf{x})$ over all shaded areas. Because the two shaded areas (in quadrants II and IV) are symmetric with $p d f_{s}(\mathbf{x}), P(" D " \mid s)$ may be obtained by integrating $2 p d f_{s}(\mathbf{x})=p d f_{A A}(\mathbf{x})+p d f_{B B}(\mathbf{x})$ over only quadrant IV. Furthermore, because the integration of $p d f_{A A}(\mathbf{x})$ over the shaded areas is the same as the integration of $p d f_{B B}(\mathbf{x}), P($ " $D$ " $\mid s)$ can be obtained by integrating $2 p d f_{A A}(\mathbf{x})$ over the shaded area in quadrant IV only. Therefore, we have

$$
P\left(“ D^{\prime \prime} \mid s\right)=2 \int_{u}^{\infty}\left[\int_{-\infty}^{v\left(x_{1}\right)} \phi\left(x_{1}-\frac{d^{\prime}}{2}\right) \phi\left(x_{1}-\frac{d^{\prime}}{2}\right) d x_{2}\right] d x_{1},
$$

which may be expressed as

$$
P(“ D ” \mid s)=2 \int_{u}^{\infty} \Phi\left(v\left(x_{1}\right)-\frac{d^{\prime}}{2}\right) \phi\left(x_{1}-\frac{d^{\prime}}{2}\right) d x_{1} .
$$

Equations B12 and B14 are used in the text.

(Manuscript received June 10, 1994; revision accepted for publication May 5, 1995.) 